\title{
AVALIAÇÃO DA MANOBRA DE REPOSICIONAMENTO DE EPLEY EM INDIVÍDUOS COM VERTIGEM POSICIONAL PAROXÍSTICA BENIGNA
}

\section{Assessing Epley's maneuver for benign paroxysmal positioning vertigo}

\author{
Viviane de Souza Pinho Costa ${ }^{(1)}$, Luciana Lozza de Moraes Marchiori (2), \\ Juliana Jandre Melo ${ }^{(3)}$, Fernando Raphael Pinto Guedes Rogério ${ }^{(4)}$, Mariana Kuss Amâncio (5), \\ Any Danielle Fontana ${ }^{(6)}$, Camila Gonçalves do Nascimento ${ }^{(7)}$
}

\begin{abstract}
RESUMO
Objetivo: avaliar os parâmetros estabilométricos após a manobra de reposicionamento de Epley em indivíduos com Vertigem Posicional Paroxística Benigna (VPPB) previamente confirmada através da manobra de Dix-Hallpike. Métodos: estudo experimental que incluiu pacientes que apresentaram VPPB com nistagmo de posicionamento à prova de Dix-Hallpike, avaliados no ano de 2008, no Ambulatório Multidisciplinar de Vertigem, e que foram submetidos à manobra de reposicionamento de Epley e avaliados quanto ao equilíbrio postural pelo exame de Estabilometria, antes e após a citada manobra. Resultados: os 13 indivíduos do gênero feminino com idade variando de 15 a 78 anos com VPPB, previamente confirmados e selecionados para a pesquisa, apresentaram melhora estatisticamente significante em parâmetros estabilométricos verificados da primeira para a segunda avaliação, confirmando melhora do equilíbrio postural, após a manobra de Epley. Conclusão: nesta pesquisa a manobra de reposicionamento de Epley mostrou-se como um procedimento de intervenção importante para a melhora das respostas do equilíbrio postural em indivíduos com VPPB avaliados pela estabilometria.
\end{abstract}

DESCRITORES: Vertigem; Equilíbrio Musculosquelético; Nistagmo

\section{INTRODUÇÃO}

A Vertigem Postural Paroxística Benigna (VPPB) constitui uma das mais frequentes

(1) Fisioterapeuta; Docente do Curso de Fisioterapia Universidade Norte do Paraná, UNOPAR, Londrina, PR, Brasil; Doutoranda pelo Programa de Enfermagem Fundamental da Escola de Enfermagem de Ribeirão Preto da Universidade de São Paulo.

(2) Fonoaudióloga; Docente do Curso de Fonoaudiologia da Universidade Norte do Paraná, UNOPAR, Londrina, PR, Brasil; Doutora em Medicina e Ciências da Saúde pela Universidade Estadual de Londrina.

(3) Fonoaudióloga; Orientadora do Curso de Fonoaudiologia da Universidade Norte do Paraná, UNOPAR, Londrina, PR, Brasil; Mestre em Fonoaudiologia pela Pontifícia Universidade Católica de São Paulo.

(4) Fisioterapeuta; Clinica Reabilitar, Londrina, PR, Brasil.

(5) Fisioterapeuta Clínica, Londrina, PR, Brasil.

(6) Fonoaudióloga; Centro Auditivo Audiolon, Londrina, PR, Brasil.

(7) Fonoaudióloga Clínica, Arapongas, PR, Brasil.

Conflito de interesses: inexistente afecções do sistema vestibular, sendo mais prevalente no sexo feminino. Caracteriza-se clinicamente por um quadro de episódios de vertigens habitualmente intensas, rotatórias, com segundos de duração e tipicamente desencadeadas, por determinados movimentos cefálicos. Dependendo de sua intensidade torna-se uma disfunção incapacitante para as pessoas acometidas para o desenvolvimento das atividades cotidianas da vida ${ }^{1}$.

A VPPB é considerada uma disfunção na orelha interna, que é composta por uma série de câmaras cheias de líquido. Dentro destas câmaras estão dispostos três canais semicirculares que são responsáveis, em parte, por sentir o movimento e manter o equilíbrio corporal. A causa da ocorrência de VPPB é o deslocamento de pequenos cristais de carbonato de cálcio, provenientes de fracionamento de estatocônias da mácula utricular, que flutuam pelo fluido da orelha interna e golpeiam os terminais nervosos sensíveis da cúpula e existentes no término de cada canal semicircular e ampolas ${ }^{1,2}$. 
Diversas são as formas de tratamento para as disfunções labirínticas, podendo ser indicados desde tratamento cirúrgico e/ou terapia medicamentosa, a reabilitação vestibular e manobras de reposicionamento das partículas de estatocônias.

Embora o tratamento da VPPB seja multiprofissional, longo e oneroso, algumas medidas vêm sendo tomadas com o intuito de torná-lo mais eficaz e menos exaustivo ao paciente. Sendo assim, a manobra de reposicionamento de Epley constitui uma forma de tratamento com boa viabilidade, baixo custo e índice de efeitos colaterais praticamente insignificantes. Esta é uma técnica simples e bem tolerada de terapia física que pode aliviar a vertigem em grande maioria dos pacientes. Segundo a literatura, cerca de $75 \%$ de pacientes ficam livres dos sintomas com uma manobra ${ }^{1,3}$.

Epley descreveu esta manobra (canalith repositioning maneuver), como sendo realizada mediante uma série de movimentos cefálicos que encaminharia os fragmentos de estatocônias de volta para o utrículo, onde seriam absorvidos ou eliminados pelo saco endolinfático ${ }^{3}$.

Em estudos recentes de revisão sistemática os autores evidenciaram que a manobra de Epley é eficaz e mantém os resultados por pelo menos três meses após sua utilização, não necessitando de variantes ou associação com outras terapias para atingir um bom resultados terapêutico da VPPB ${ }^{1}$.

Os pacientes com VPPB geralmente relatam que a vertigem é desencadeada quando estão deitados, mudam de posição na cama, inclinam o tronco para frente ou olham para cima. As situações comuns em que a vertigem é provocada incluem levantarse da cama, praticar jardinagem, lavar os cabelos no chuveiro, ir ao dentista ou ao salão de beleza. Outras queixas associadas à VPPB incluem problemas de equilíbrio que podem durar horas ou dias após a interrupção do episódio, assim como sensações mais vagas, como a tontura ou uma sensação de flutuar ${ }^{1,2}$.

O mapeamento do equilíbrio em diferentes posições da base de estabilidade pode ser útil para avaliar o desempenho do sistema de controle postural em controlar o equilíbrio em situações extremas em que o corpo humano possa cair ${ }^{4}$.

Apesar da constatação da melhora clínica dos pacientes após a realização da manobra de Epley, relatada em vários estudos, há escassez de dados na literatura, que quantifiquem a melhora desta manobra. Sendo assim, é de suma importância a avaliação da mudança no equilíbrio constatado pela estabilometria, após a manobra de Epley, tanto para o prognóstico como para o tratamento de pacientes com VPPB. Isto devido à estabilometria ou estabilografia ou estatocinesiografia ser um importante método para quantificar a integridade dos sistemas de controle postural por se consistir em um recente método de avaliação do equilíbrio postural estático humano ${ }^{4}$.

Então, o parâmetro comumente analisado para a quantificação desta variável em postura estática e ortostática é a oscilação do centro de pressão, sendo esse uma medida de deslocamento e dependente do centro de gravidade do corpo. A oscilação do centro de gravidade representa uma oscilação do corpo e a oscilação do centro de pressão representa uma resposta neuromuscular ao balanço do centro de gravidade ${ }^{4}$.

Uma vez que as manobras mecânicas de reabilitação vestibular são utilizadas como opções terapêuticas para o tratamento da VPPB por proporcionar o reposicionamento das estatocônias de volta ao utrículo, por meio de uma sequência de movimentos cefálicos, o objetivo deste estudo foi verificar a resposta dos parâmetros estabilométricos à manobra de reposicionamento de Epley em indivíduos com VPPB previamente confirmada através da manobra de Dix-Hallpike.

\section{MÉTODOS}

Trata-se de um estudo quantitativo, descritivo e experimental. A amostra constituiu-se por 13 indivíduos do gênero feminino, com VPPB, selecionados pelo método de amostragem não probabilístico e por conveniência. Houve a inclusão de pacientes com diagnóstico de VPPB a partir de sua história e do exame físico, sem perdas auditivas e, que apresentaram a presença de nistagmo de posicionamento à prova de Dix-Hallpike, avaliados no ano de 2008 no Ambulatório de Vertigem da Universidade Norte do Paraná (UNOPAR), e com as vestibulopatias centrais descartadas, e sem queixas ortopédicas ou fraturas recentes nos últimos três meses nos membros inferiores.

A confirmação do diagnóstico de VPPB entre os participantes do estudo foi realizado o teste de Dix-Hallpike, na qual a prova é considerada positiva se houver aparecimento de nistagmo com os olhos abertos, acompanhado de vertigem ${ }^{1}$.

Os procedimentos de intervenção ocorreram em três etapas: inicialmente os pacientes foram submetidos ao teste estabilométrico para a avaliação do equilíbrio corporal postural e as oscilações do centro de pressão, e então, num segundo momento foi aplicada a manobra de reposicionamento de Epley como único procedimento terapêutico para a VPPB, e por último, refeito o segundo teste estabilométrico para avaliação das diferenças de oscilação do centro de pressão pertinentes aos resultados da manobra. 
A manobra de reposicionamento Epley foi realizada com a cabeça do paciente sendo rodada $45^{\circ}$ para o lado comprometido. Em seguida, o paciente é rapidamente deitado mantendo a posição da cabeça em relação ao tronco, sendo o segmento cefálico rapidamente rodado em duas etapas de $90^{\circ}$ com o paciente sendo então levantado rapidamente ${ }^{3}$.

O exame estabilométrico foi realizado com uso da Plataforma Eclipse $\mathbf{3 0 0 0}$ da marca Guy-Capron SA, França, com superfícies de $40 \times 40 \mathrm{~cm}$, para a realização de baropodometria computadorizada com o intuito de verificar assimetrias, desníveis e desvios posturais, e correlacionar os desvios e assimetrias posturais com o equilíbrio corporal postural, em ambiente tranquilo, com os indivíduos em apoio bipodal, com pés descalços, com a base de apoio posicionada em largura confortável, os braços soltos e relaxados ao lado do corpo e com o segmento cefálico posicionado horizontalmente ao plano do solo. O teste foi realizado na condição experimental, olhos abertos, solicitando-se que os indivíduos observassem um ponto localizado a 2 metros de distância na parede a sua frente e a altura de seus olhos.

A avaliação estabilométrica pré e pós a realização da manobra de Epley foi responsável por identificar os parâmetros: largura de oscilação do centro de pressão no eixo Y (ântero-posterior); largura de oscilação do centro de pressão no eixo $X$ (desvio lateral); área total de deslocamento do centro de pressão; velocidade média quadrática do deslocamento do centro de pressão.

Todos os indivíduos participaram voluntariamente após assinarem o termo de consentimento livre e esclarecido do projeto de pesquisa aprovado pelo Comitê de Ética em Pesquisa da própria Instituição, com número de protocolo PP/0063/08.

A análise estatística foi realizada através do programa Bioestat 4.0, onde inicialmente, aplicouse o teste de normalidade de Shapiro-Wilk para verificar a homogeneidade dos dados e, posteriormente, para comparação dos escores referentes às variáveis estabilométricas pré e pós a aplicação da manobra de reposicionamento, realizado pelo Teste de Wilcoxon, com significância de $p<0,05$.

\section{RESULTADOS}

A população estudada foi constituída por 13 mulheres com idade variando de 15 a 78 anos, selecionados por apresentarem sinais clínicos da VPPB, confirmados pela manobra de Dix-Hallpike.

Os valores médios e o desvio padrão (DP) do grupo, antes da manobra de Epley, foram: largura de deslocamento do centro de pressão no sentido ântero-posterior foi de 20,38mm $(\mathrm{DP} \pm 3,97)$, a largura de deslocamento do centro de pressão no sentido látero-lateral 9,92mm ( $\mathrm{DP} \pm 16,27)$, a área de deslocamento do centro de pressão foi $147,92 \mathrm{~mm}$ $(\mathrm{DP} \pm 4.02)$ e a velocidade média quadrática de $10,54 \mathrm{~mm} / \mathrm{s}$ (DP \pm 2.01 ).

Após a manobra de repocisionamento de Epley em todos os indivíduos os escores das variáveis estabilométricas apresentaram-se reduzidos: largura de deslocamento do centro de pressão no sentido ântero-posterior reduziu para $06 \mathrm{~mm}\left(\mathrm{DP}_{ \pm}\right.$ $7,25 \mathrm{~mm}$ ), bem como, a largura de deslocamento do centro de pressão no sentido látero-lateral com $3.84 \mathrm{~mm}(\mathrm{DP} \pm 4.65)$, a área de deslocamento do centro de pressão $22,30 \mathrm{~mm}(\mathrm{DP} \pm 4.56)$ e velocidade média quadrática foi de $5,39 \mathrm{~mm} / \mathrm{s}(\mathrm{DP} \pm 4.13)$. Em relação aos escores das variáveis estabilométricas obtidas antes e após a manobra de reposicionamento de Epley, observou-se diferença estatisticamente significante em relação largura de oscilação do centro de pressão na direção ântero-posterior $(p=0,015)$, largura de oscilação do centro de pressão na direção látero-lateral ( $p=0,003)$; a área total de deslocamento do centro de pressão $(p=0,015)$ e a velocidade média quadrática do deslocamento do centro de pressão com $p=0,17$, demonstrando, por conseguinte que a manobra não surtiu efeito clínico significante somente na variável velocidade média quadrática.

Os pacientes ressaltaram melhora do quadro clínico relacionado às intercorrências de vertigem rotatória na VPPB ao final de todas estas intervenções, bem como após um segmento de um mês em entrevista pessoal com todos os participantes do estudo.

\section{DISCUSSÃO}

Para que haja a manutenção da postura e do equilíbrio é necessária à integridade dos sistemas visual, vestibular e sensório-motor, sendo que alterações nestas funções são frequentemente encontradas em pacientes com queixa de tontura que pode ser rotatória. A tontura quando rotatória é denominada vertigem e geralmente é ocasionada por alterações na parte vestibular do ouvido interno, ocasionando o desconforto durante a movimentação do indivíduo quanto à mudança de posição cefálica ${ }^{1,4}$.

A principal manobra diagnóstica para a VPPB é a prova de Dix-Hallpike, que por meio do posicionamento de cabeça nas diversas posições do espaço, para as duas direções direita e esquerda, e observar se desencadeia algum sintoma ou sinal labiríntico como a vertigem, náusea e o nistagmo ${ }^{1}$. 
Quanto à manobra de reposicionamento de DixHallpike, como o citado na literatura da área, constitui-se em método simples e eficiente na detecção da VPPB e deve ser incluída na propedêutica usual para avaliação de pacientes queixosos de vertigem, além de permitir a imediata execução de um método simples, rápido, fácil, de baixo custo e além de tudo sustentado por um bom, e não ótimo, porém crescente suporte de evidências científicas ${ }^{5}$.

Os pacientes apresentados com história clínica condizente com o quadro de VPPB pela presença de uma tontura desencadeada por movimentos cefálicos apresentaram a condição básica para o diagnóstico, ou seja, o nistagmo desencadeado pela manobra de Dix-Hallpike, como o encontrado na literatura da área, realmente tiveram benefício postural estatisticamente significante após a realização da manobra de Epley, tal quais os casos encontrados na literatura da área, que colocam que a manobra de Epley vem apresentando excelentes índices terapêuticos de melhora clínica ${ }^{1,6-10}$.

Os benefícios da manobra de reposicionamento de Epley mais uma vez foram verificados neste estudo, porém, por meio da utilização e avaliação estabilométrica, que por ser um método quantitativo, pode ser utilizada para avaliar um mesmo paciente, trazendo dados valiosos a respeito de sua melhora postural subseqüente a manobra de reposicionamento de Epley.

A Estatocinesiometria faz parte da Posturografia que permite avaliar quantitativamente a componente vestíbulo-espinhal do equilíbrio corporal e tem sido utilizada por vários autores em pesquisa e avaliação clínica por fornecer medidas da função vestíbulo-espinhal, proporcionando informações complementares indispensáveis para avaliação de pacientes com tontura e analisando as interações sensoriais ${ }^{11}$. Nesta pesquisa, este método se mostrou bastante eficiente para o fornecimento da efetividade da manobra de Epley em relação à melhora do equilíbrio postural da população estudada.

Alguns dos trabalhos na área demonstram índices de eficácia da manobra de reposição canalítica pouco expressivos quanto à melhora dos sintomas por um longo período, bem como fracas evidências quando comparado com outros recursos terapêuticos para VPPB. Porém, outros estudos mostram grande importância na reposição canalítica 9,12,13.

Em um estudo com 40 sujeitos, sendo 20 submetidos à manobra de reposicionamento de Epley e 20 submetidos a placebo, verificou-se após uma semana que todos os pacientes apresentaram negatividade na manobra de Dix-Hallpike enquanto apenas $30 \%$ do grupo controle apresentou negatividade durante a citada manobra de avaliação ${ }^{14}$.
Em estudo a respeito da eficácia da manobra de Epley, compararam a citada manobra e placebo. Os pacientes foram reavaliados no período de um mês através da manobra de Dix-Hallpike e através de diários sendo observadas repostas negativas ao teste em $88,9 \%$ no grupo submetido à citada manobra e $26,7 \%$ no placebo com melhora foi referida em $61,1 \%$ no grupo tratado enquanto $20 \%$ no placebo ${ }^{15}$.

Ao averiguarem a necessidade do agrupamento de evidências científicas que demonstrassem uma mensuração da eficácia das manobras propostas para o tratamento da VPPB, realizaram uma revisão sistemática da literatura para avaliar a eficácia das manobras liberatórias em pacientes com o este diagnóstico e concluíram que a cinesioterapia, através da manobra de Epley, é eficaz para o tratamento da VPPB quando comparado a placebo e/ou tratamento medicamentoso isolado e/ou nãointervenção ${ }^{5}$.

Outros autores descreveram também os benefícios proporcionados pela manobra de Epley, no qual pela avaliação de dois grupos de pacientes, perfazendo um total 123 pessoas, identificaram a redução significativa do aparecimento de episódios de nistagmo no grupo tratado com a intervenção da manobra em quatro momentos numa mesma terapia, em relação ao grupo que a realizou uma única vez por sessão durante algumas semanas, considerando que apenas o procedimento de tratamento com esta manobra de reposicionamento pode ser benéfico para a redução dos sinais e sintomas clíni$\cos$ da VPPB ${ }^{16}$. Os resultados demonstraram que a realização apenas da manobra pode ser suficiente para o tratamento das pessoas com VPPB, sem necessitar de variações e associações de outras terapias para atingir um bom resultado terapêutico como visto em outros trabalhos na mesma área.

Portanto, estudos, em várias áreas, têm sido desenvolvidos de modo a contribuir para a verificação do equilíbrio de modo geral e mais especificamente associado ao quadro de vertigem, abordando e visando melhoria da qualidade de vida 17-22. Esta atitude terapêutica certamente ajudará os muitos pacientes, que pela tontura e mais especificamente as vertigens, deliberadamente restringem suas atividades cotidianas e sociais da vida, com o intuito de reduzir o risco de aparecimento destes sintomas desagradáveis e assustadores, e para evitar o embaraço social e o estigma que eles podem causar.

\section{CONCLUSÃO}

Em relação ao objetivo deste estudo foi verificado que as respostas finais dos parâmetros 
estabilométricos, após a manobra de reposicionamento de Epley em indivíduos com VPPB, resultaram em comprovação da efetividade em relação ao equilíbrio postural de forma imediata, proporcionando melhor estabilidade em relação às oscilações ocorridas no corpo anteriormente à sua aplicação.

\begin{abstract}
Purpose: to evaluate the stabilometry parameters after Epley's maneuver in individuals with BPPV previously confirmed through Dix-Hallpike maneuver. Methods: an experimental study which included patients with BPPV with positional nystagmus taking Dix-Hallpike's test and assessed in 2008, at the Multidisciplinary Vertigo Ambulatory, and who were also submitted to Epley's maneuver and assessed as for their postural balance through a stabilometry exam, both before and after such maneuver. Results: the 13 female subjects with ages ranging from 15 to 78 years with previously confirmed BPPV, who were selected for the research, showed a significant statistically improvement regarding their postural balance after Epley's maneuver in all analyzed parameters as for both exams. Conclusion: in this research the Epley's maneuver was shown as a procedure of important intervention for improving the answers of postural balance in individuals with BPPV evaluated by the stabilometry.
\end{abstract}

KEYWORDS: Vertigo; Musculoskeletal Equilibrium; Nystagmus

\section{REFERÊNCIAS}

1. Burlamaqui JC, Campos $\mathrm{CAH}$, Mesquita Neto O. Manobra de Epley para Vertigem Postural Paroxística Benigna: revisão sistemática. Acta Otorrinolaringol. 2006; 24(1):38-45.

2. Dix MR, Hallpike CS. The pathology, symptomatology and diagnosis of certain common disorders of the vestibular system. Ann Otol Rhinol Laryngol. 1952; 45(6):341-54.

3. Epley JM. The canalith reposiotining procedure for treatment of benign paroxysmal positional vertigo. Otolaryngol Head Neck Surg. 1992; 107(3):399-404. 4. Duarte M. Análise estabilográfica da postura ereta humana quase estática. [livre-docência]. São Paulo (SP): Universidade de São Paulo; 2000.

5. Teixeira LJ, Machado JNP. Manobras para o tratamento da vertigem posicional paroxística benigna: revisão sistemática da literatura. Rev Bras Otorrinolaringol. 2006; 72(1):130-9.

6. Norré ME, Forrez G. Posture testing (posturography) in the diagnosis of peripheral vestibular pathology. Arch Otorhinolaryngol. 1986; 243(3):186-9.

7. Woodworth BA, Gillespie MB, Lambert PR. The canalith repositioning procedure for benign positional vertigo: a meta-analysis. Laryngoscope. 2004; 114(7):1143-6.

8. Steenerson RL, Cronin GW, Marbach PM. Effectiveness of treatment techniques in 923 cases of benign paroxysmal positional vertigo. Laryngoscope. 2005; 115(2):226-31. http://dx.doi. org/10.1097/01.mlg.0000154723.55045.b5
9. Helminski JO, Janssen I, Kotaspouikis D, Kovacs K, Sheldon P, McQueen K, et al. Strategies to prevent recurrence of benign paroxysmal positional vertigo. Arch Otolaryngol Head Neck Surg. 2005; 131(4):344-8.

10. Richard W, Bruintjes TD, Oostenbrink P, van Leewen RB. Efficacy of the Epley maneuver for posterior canal BPPV: a long-term, controlled study of 81 patients. Ear Nose Throat J. 2005; 84(1):22-5. 11. Marchiori LLM, Rego Filho EA. Queixa de vertigem e hipertensão arterial. Rev CEFAC. 2007; 9(1):116-21. http://dx.doi.org/ 10.1590/S1516-18462007000100015

12. Hansson EE, Mansson NO, Hakansson A. Benign Paroxysmal Positional Vertigo among elderly patients in primary health care. Gerontol. 2005; 51(6):386-9. http://dx.doi.org/10.1159/000088702

13. Inukai K, Koizuka I, Takahashi S. Head-tilting stabilometry in patients with benign paroxysmal positional vertigo. Auris Nasus Larynx. 2008; 35(1):31-5. http://dx.doi.org/10.1016/j.anl.2007. 06.007 14. Sridhar S, Panda N, Raghunathan M. Efficacy of particle repositioning maneuver in BPPV: a prospective study. Am J Otolaryngol. 2003; 24(6):355-60.

15. Lynn S, Pool A, Rose D, Brey R, Suman V. Randomized trial of the canalith repositioning procedure. Otolaryngol Head Neck Surg. 1995; 113(6):712-20.

16. Korn GP, Dorigueto RS, Ganança MM, Caovilla $\mathrm{HH}$. Repeated Epley's maneuver in the same session in benign positional paroxysmal vertigo. Rev Bras Otorrinolaringol. 2007; 73(4):533-9. 
17. Lorin P. Benign paroxysmal positional vertigo of the anterior semicircular canal: clinical aspects and treatment. Rev Laryngol Otol Rhinol (Bord). 2005; 126(4):263-6.

18. Neuhauser HK, Radtke $A$, von Brevern $M$, Feldmann M, Lezius $\mathrm{F}$, Ziese $\mathrm{T}$, et al. Migrainous vertigo: prevalence and impact on quality of life. Neurology. 2006; 67(6):1028-33.

19. Neuhauser HK, Radtke A, von Brevern M, Lezius $F$, Feldmann $M$, Lempert $T$. Burden of dizziness and vertigo in the community. Arch Int Med. 2008; 168(19):2118 -24.
20. Neuhauser HK, von Brevern $M$, Radtke $A$, Lezius F, Feldmann M, Ziese T, et al. Epidemiology of vestibular vertigo. A neurotologic survey of the general population. Neurology. 2005; 65:898-904. 21. Levandowski MA, Bueno VK, Marchiori LLM, Melo JJ. Vertigem no idoso: relato de caso. Rev. CEFAC. 2008; 10(4): 588-91. http://dx.doi. org/10.1590/S1516-18462008000400019

22. Sauvage JP, Aubry K, Codron S. Benign paroxysmal positional vertigo of the horizontal and superior semicircular canals. Rev Laryngol Otol Rhinol (Bord). 2005; 126(4):257-62.

DOI: 10.1590/S1516-18462010005000023

RECEBIDO EM: 14/04/2009

ACEITO EM: 30/09/2009

Endereço para correspondência:

Luciana Lozza de Moraes Marchiori

Av. Paris, 675

Londrina - PR

CEP: 86041-100

E-mail: luciana.marchiori@londrina.net 